\title{
Medical care emerging challenges for older people during early COVID-19 pandemic
}

\author{
George Soulis ${ }^{1} \cdot$ Marco Inzitari $^{2,3}$
}

Published online: 14 February 2022

(c) The Author(s), under exclusive licence to European Geriatric Medicine Society 2022

During the COVID-19 pandemics, the majority of healthcare services and professionals, especially those working with older people, have been facing follow-up visits' omissions and cancellations, sourcing either from patients and families or from the providers. Arguments, in most cases, refer to the perceived threat of COVID-19 infection and the easiness of COVID-19 transmission.

The intensity of COVID-19 pandemic waves has put extreme pressure on the healthcare systems Worldwide. In most cases, these systems were poorly prepared and sometimes have not prioritized the care of users with health problems and needs different from COVID-19. The article by Schuster et al., published in a previous issue of the journal and based on the consolidated, large epidemiological cohort of the Longitudinal Amsterdam Study of Aging (LASA), emphatically reveals a hidden side of the stress that health care systems suffered during COVID-19 pandemics, a real problem that emerged and relates to the incomplete service provision, in this case to the particularly vulnerable risk group of older people. This was either due to patient initiative or to the healthcare provider and, surprisingly, concerned more often the people with multiple morbidities. According to this important work, this medical care cancellation or avoidance was relatively widespread, as it affected one third of the older population included in the study, in both community and outpatient hospital settings [1].

Other reports have shown that this reduced activity during the pandemic, in particular in its first waves, did not

Marco Inzitari

minzitari@perevirgili.cat

1 Department of Public and Community Health, School of Public Health, University of West Attica, Athens, Greece

2 REFiT Barcelona Research Group, Parc Sanitari Pere Virgili and Vall d'Hebron Institute of Research (VHIR), Edifici Montseny 0, c Esteve Terrades 30, 08023 Barcelona, Spain

3 Faculty of Healthcare Sciences, Universitat Oberta de Catalunya (UOC), Barcelona, Spain only happen in outpatients environments, but also in acute care, even for serious events such as a stroke [2] or the older adults rehabilitation setting [3].

This decrease in service use might be likely due to multiple factors, which, as mentioned, might rely on patients' and families' psychological representations (fear of the infection and anxiety or fatigue), which also contributed negatively to older people's activity and mobility [4]). On the other hand, many healthcare services were reorganized, with a consequent minor accessibility to non-COVID-19 resources, linked to service reduction and restrictions related to protocols aiming to reduce the transmission, but also in some cases, due to the shortage of professionals, absorbed to face the massive needs in expanded acute services. A change in the format of the provision was observed, shifting towards modalities which might limit the access to older adults, in particular older ones or with lower education [5] and possibly those more disabled or lacking social support.

System response parameters such as resilience stability and robustness were challenged (for review see [6])). Telemedicine use expansion may be a way to deal with the healthcare system pressure, although a balance between telemedicine and in person visits should be reached whenever the situation is stabilized. As mentioned, there are some thoughts mainly concerning issues such as inequity, lack of standardization, age friendliness for older people, difficulties faced by older people with physical and cognitive difficulties, but generally telehealth emerged as a useful option that needs to be optimized especially when assessing older people [7]. Anyway, more work is needed to tailor solutions which might better deal with heterogeneity of the older adult population, and a user-participation research and innovation approach is warranted in this sense.

As a conclusion, the study by Schuster et al. strongly urges the healthcare systems to recognize delays in health care provision to older people to design-together with stakeholders as healthcare providers, professionals, administrators and end users-specific plans to promote an active 
recuperation of the lost ground. This can possibly be based on a case-mix stratification to prioritize the most urgent situations and offer to everyone the most adequate care in the most suitable format and time, as well as in the most appropriate environment. In this sense, the changes promoted inevitably by the pandemics, such as virtual care options and prioritization strategies, should be integrated in the gradual health care system adaptation, in this evolving situation. Tomorrow's health care services have to take into account both the potential of a new pandemic and the impact of such a scenario to the management of chronic diseases and should design action plans that will better absorb the stress and make the systems more resilient.

\section{Declarations}

Conflict of interest The authors declare no conflcts of interest related to this work.

Ethical approval This editorial did not need approval by Ethics Committee or Institutional Review Board.

Statement for human and animal rights This work did not involve experiments or novel data collection with humans or animals.

Informed consent This editorial did not involve any participating human subject, so that the informed consent was not necessary.

\section{References}

1. Schuster NA, de Breij S, Schaap LA et al (2021) Older adults report cancellation or avoidance of medical care during the
COVID-19 pandemic: results from the Longitudinal Aging Study Amsterdam. Eur Geriatr Med 12:1075-1083. https://doi.org/10. 1007/s41999-021-00514-3

2. Rudilosso S, Laredo C, Vera V et al (2020) Acute stroke care is at risk in the era of COVID-19: experience at a comprehensive stroke center in Barcelona. Stroke 51(7):1991-1995. https://doi. org/10.1161/STROKEAHA.120.030329

3. Prvu Bettger J, Thoumi A, Marquevich V et al (2020) COVID19: maintaining essential rehabilitation services across the care continuum. BMJ Glob Health 5(5):e002670. https://doi.org/10. 1136/bmjgh-2020-002670

4. Pérez LM, Castellano-Tejedor C, Cesari M et al (2021) Depressive symptoms, fatigue and social relationships influenced physical activity in frail older community-dwellers during the Spanish lockdown due to the COVID-19 pandemic. Int J Environ Res Public Health 18(2):808. https://doi.org/10.3390/ijerph18020808

5. Ali MA, Alam K, Taylor B, Ashraf M (2021) Examining the determinants of eHealth usage among elderly people with disability: the moderating role of behavioural aspects. Int J Med Inform 149:104411. https://doi.org/10.1016/j.ijmedinf.2021.104411

6. Xueming L, Daqing L, Manqing $M$ et al, Network resilience. arXiv:2007.14464v1 [q-bio.QM]

7. Doraiswamy S, Jithesh A, Mamtani R et al (2021) Telehealth use in geriatrics care during the COVID-19 pandemic-a scoping review and evidence synthesis. Int J Environ Res Public Health 18(4):1755. https://doi.org/10.3390/ijerph18041755

Publisher's Note Springer Nature remains neutral with regard to jurisdictional claims in published maps and institutional affiliations. 